\title{
From Rindler Fluid to Dark Fluid on the Holographic Cutoff Surface
}

\author{
Rong-Gen Cai ${ }^{1,2}$, Gansukh Tumurtushaa ${ }^{3,4}$ and Yun-Long Zhang ${ }^{5,6 \star}$
}

1 Institute of Theoretical Physics, Chinese Academy of Sciences(ITP-CAS) and School of

Physical Sciences, University of Chinese Academy of Sciences, Beijing, China

2 School of Fundamental Physics and Mathematical Sciences, Hangzhou Institute for

Advanced Study, University of Chinese Academy of Sciences, Hangzhou, China

3 Center for Quantum Spacetime(CQUeST), Sogang University, Seoul, Korea

4 Center for Theoretical Physics of the Universe, Institute for Basic Science, Daejeon, Korea

5 Center for Gravitational Physics, Yukawa Institute for Theoretical Physics(YITP), Kyoto

University, Kyoto, Japan

6 National Astronomy Observatories, Chinese Academy of Science, Beijing, China

$\star$ [zhangyunlong001@gmail.com]

4th International Conference on Holography,

String Theory and Discrete Approach

Hanoi, Vietnam, 2020

doi:10.21468/SciPostPhysProc.4

\begin{abstract}
As an approximation to the near horizon regime of black hols, the Rindler fluid was proposed on an accelerating cutoff surface in the flat spacetime. The concept of the Rindler fluid was then generalized into a flat bulk with the cutoff surface of the induced de Sitter and FRW universe, such that an effective description of dark fluid in the accelerating universe can be investigated.
\end{abstract}

(c) (1) Copyright A. Bee et al.

This work is licensed under the Creative Commons

Attribution 4.0 International License.

Published by the SciPost Foundation.
Received ??-??-20??

Accepted ??-??-20??

Published ??-??-20??

doi:10.21468/SciPostPhysProc.4.??

\section{Contents}

2 Dark Fluid on Holographic Cutoff 2

3 Modified Friedmann equation 3

$\begin{array}{lll}4 & \text { Summary } & 4\end{array}$

References $\quad 5$ 


\section{Introduction}

The origin and properties of the dark fluid, mainly including the dark energy and dark matter, are still mysterious in the current universe. The model of Lambda Cold Dark Matter $(\Lambda \mathrm{CDM})$ treats dark energy as the cosmological constant and dark matter as the collision-less particles, and explains the cosmic evolution and large-scale structures well. However, the tension between local measurements of the Hubble constant and the Planck's observation based on $\Lambda \mathrm{CDM}$ model becomes more important $[1,2]$. Besides, the dark matter particles have not been detected directly. Thus, alternative models of the dark fluid such as modified gravity need to be reconsidered. One recent example is the emergent gravity by Verlinde [3], which is inspired by the volume law correction to the entropy on a holographic screen, whereas the Einstein gravity is related to the area law [4].

So is there a model which can unify these two scenarios of dark fluid and modified gravity? In this article, we show that a holographic model of the emergent dark universe (hEDU) can naturally realize the duality between the dark fluid in $(3+1)$-dimension and a modified gravity in (4+1)-dimension. We consider that the dark fluid in the universe emerges as the holographic stress-energy tensor on the hypersurface in one higher dimensional flat bulk [5,6]. After adding the localized stress-energy tensor $T_{\mu \nu}$ on the hypersurface with intrinsic metric $g_{\mu \nu}$ and extrinsic curvature $\mathcal{K}_{\mu \nu}$, the induced Einstein field equations on the holographic screen are modified as

$$
R_{\mu \nu}-\frac{1}{2} g_{\mu \nu} R=\kappa_{4}\left(T_{\mu \nu}+\langle\mathcal{T}\rangle_{\mu \nu}^{d}\right),
$$

where $\langle\mathcal{T}\rangle_{\mu \nu}^{d}$ denotes the induced Brown-York stress-energy tensor [7],

$$
\langle\mathcal{T}\rangle_{\mu \nu}^{d} \equiv \frac{1}{\kappa_{4} L}\left(\mathcal{K}_{\mu \nu}-\mathcal{K} g_{\mu \nu}\right)
$$

Here, $\kappa_{4}=8 \pi G_{4} / c^{4}$ is the Einstein constant and the length scale $L=\kappa_{5} / \kappa_{4}$ is related to the positive cosmological constant $\Lambda=3 / L^{2}$. At the cosmological scale, we assume that $T_{\mu \nu}$ only includes the components of normal matter, and $\langle\mathcal{T}\rangle_{\mu \nu}^{d}$ represents the total dark components in our universe, such as dark energy and dark matter. The stress-energy tensor $\langle\mathcal{T}\rangle_{\mu \nu}^{d}$ as we formulated is similar to the Verlinde's elastic response of emergent gravity [3], in the way that it will back react on the background geometry.

The using of the Brown-York stress-energy tensor in (2) is inspired by the Wilsonian renormalization group (RG) flow approaches of fluid/gravity duality [8-14]. Where the holographic stress-energy tensor on the holographic cutoff surface is identified with the stress energy tensor of the dual fluid directly. When taking the near horizon limit, one can reach the so-called Rindler fluid [15-22], which is a new perspective on the membrane paradigm of black holes, where the Brown-York stress-energy tensor is used.

\section{Dark Fluid on Holographic Cutoff}

To see more clearly how the Einstein equation (1) works, it is interesting to consider a de Sitter hypersurface as the holographic screen in flat spacetime firstly. Then the dual stress tensor could contribute to the dark energy as $\langle\mathcal{T}\rangle_{\mu \nu}^{\Lambda}=-\left(\rho_{c} \tilde{\Omega}_{\Lambda}\right) g_{\mu \nu}$. After adding the baryonic matter with typical 4-velocity $u_{\mu}$ and stress-energy tensor $T_{\mu \nu}=\left(\rho_{c} \tilde{\Omega}_{B}\right) u_{\mu} u_{v}$ on the screen, both of dark matter and dark energy can be described by the stress-energy tensor of holographic dark fluid $\langle\mathcal{T}\rangle_{\mu \nu}=\langle\mathcal{T}\rangle_{\mu \nu}+\langle\mathcal{T}\rangle_{\mu \nu}^{D}$, where $\langle\mathcal{T}\rangle_{\mu \nu}^{D}=\left(\rho_{c} \tilde{\Omega}_{D}\right)\left[\left(1+\tilde{w}_{D}\right) u_{\mu} u_{v}+\tilde{w}_{D} g_{\mu \nu}\right]$ and $\tilde{w}_{D}$ is the 
equation of state of the emergent dark matter. From the Hamiltonian constraint equation in higher dimensional spacetime, an interesting relation between these components can be derived [5],

$$
\text { hEDU : } \quad \tilde{\Omega}_{D}^{2}=\frac{\tilde{\Omega}_{\Lambda}}{2\left(1+3 \tilde{w}_{D}\right)}\left[\tilde{\Omega}_{D}\left(1-3 \tilde{w}_{D}\right)-\tilde{\Omega}_{B}\right] .
$$

Once setting $\tilde{w}_{D}=0$, we can compare (3) with the $\Lambda$ CDM parameterization and it is straightforward to take the values from the observational data by Planck collaboration [23]. The toy constraint relation (3) can be satisfied within the margin of error $\Omega_{D}^{2}-\frac{1}{2} \Omega_{L}\left(\Omega_{D}-\Omega_{B}\right) \lesssim 1 \%$. After considering $1 \simeq \Omega_{L}+\Omega_{B}+\Omega_{D}$, we also have $\Omega_{B} \simeq \Omega_{D}-3 \Omega_{D}^{2}-\Omega_{B}^{2}$. In order to see this relation more clearly we plot it in Fig. 1 , together with Verlinde's relation $\Omega_{B}=\frac{3}{4} \Omega_{D}^{2}$.

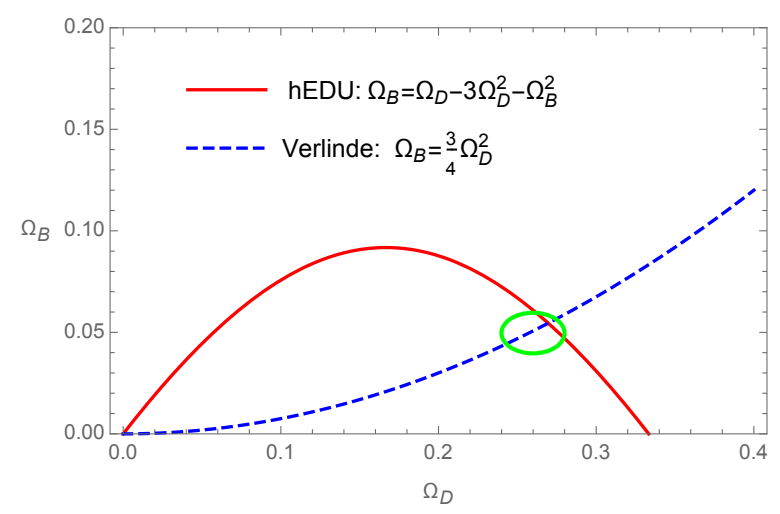

Figure 1: The schematic diagram of the relations between the components of baryonic matter $\Omega_{B}$ and dark matter $\Omega_{D}$ in the present universe. The green circle indicates the rough regime from the observation with $\Omega_{B} \simeq 0.05 \pm 0.01, \Omega_{D} \simeq 0.26 \pm 0.02$.

\section{Modified Friedmann equation}

The consistent embedding of a Friedmann-Lemaitre-Robertson-Walker (FLRW) universe in $4+1$ dimensional flat spacetime has been studied in [24,25]. In the spirit of the membrane paradigm [26,27], we remove half part of the bulk spacetime, which can be effectively replaced by the holographic stress tensor $\langle\mathcal{T}\rangle_{\mu \nu}^{d}$ in (2). The energy density and pressure in $\langle\mathcal{T}\rangle_{\mu v}^{d}$ are calculated to be $\rho_{d}(t)=\rho_{c} \sqrt{\Omega_{L}} \sqrt{\frac{H(t)^{2}}{H_{0}^{2}}+\frac{\Omega_{I}}{a(t)^{4}}}$, where the critical density and other parameters are given by $\rho_{c}=\frac{3 H_{0}^{2} M_{P}^{2}}{\hbar c}, \Omega_{L}=\frac{c^{2}}{L^{2} H_{0}^{2}}$ and $\Omega_{I} \equiv \frac{I c^{2}}{L^{2} H_{0}^{2}}$. Considering the relation between the redshift $z$ and the scale factor via $a(t) / a\left(t_{0}\right)=1 /(1+z)$, we arrive at the normalized Hubble parameters $H(z) / H_{0}$ in terms of the redshift $z$, which is the modified Friedmann equation in the hEDU model,

$$
\frac{H(z)^{2}}{H_{0}^{2}}=\frac{\Omega_{L}}{2}+\Omega_{m}(1+z)^{3}+\Omega_{r}(1+z)^{4}+\frac{\Omega_{L}}{2} \sqrt{1+\frac{4}{\Omega_{L}}\left[\Omega_{m}(1+z)^{3}+\left(\Omega_{r}+\Omega_{I}\right)(1+z)^{4}\right]} .
$$

Notice here that at the current universe $z=0$, we have $1=\Omega_{m}+\Omega_{r}+\sqrt{\Omega_{L}\left(1+\Omega_{I}\right)}$, and we will consider the fact that the radition compoents $\Omega_{r} \ll 1$. By setting $\Omega_{I}=0$, we can recover the usual Friedmann equation of the self-accelerating branch of the DGP braneworld model (sDGP) $[28,29]$. When $\Omega_{I} \ll 1$, the behavior of $\Omega_{I}(1+z)^{4}$ is more like the dark radiation [30]. However, in this hEDU model, $\Omega_{I} \gg \Omega_{r}$ turns out not to be so small, such 
that the whole dark sector, including dark energy and apparent dark matter, is expected to be included in the holographic dark fluid [5]. In Fig. 2, we plot the equation of state parameter of the holographic dark fluid $\tilde{w}_{d}(z)$ in terms of the redshift $z$, as well as the $\tilde{w}_{D}(z)$ of apparent dark matter where the effective components of cosmological constant $\Lambda$ has been deducted.
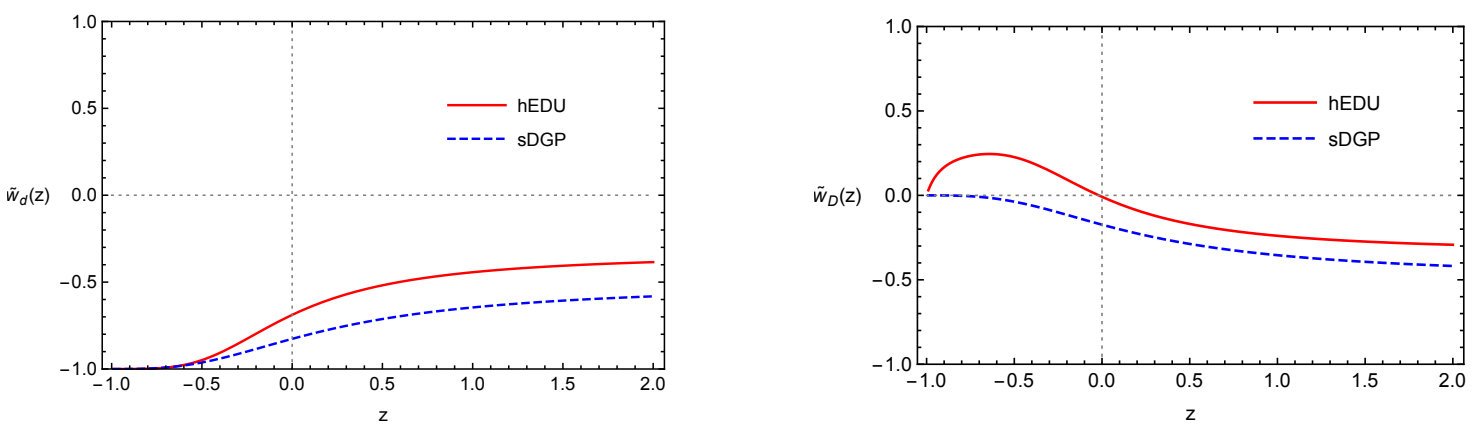

Figure 2: Left: the equation of state of the holographic dark fluid $\tilde{w}_{d}(z)$ in terms of the redshift $z$. Right: the equation of state of apparent dark matter $\tilde{w}_{D}(z)$, after deducting an effective cosmological constant. We adopt the following value for sDGP: $\Omega_{I}=0, \Omega_{m}=0.21$ [31] and hEDU: $\Omega_{I}=0.4, \Omega_{m}=0.04$ [6].

In [6], the Markov-chain Monte Carlo (MCMC) sampling analysis together with the observational data of Type Ia supernovae (SNIa) and the direct measurement of Hubble constant $H_{0}$ [32] are employed. The two-dimensional observational contours are plotted in Fig. 3, with the 1-3 $\sigma$ confidence contours for various parameters in the hEDU model [6]. The best-fit values turn out to be $\Omega_{I}=0.43 \pm 0.13$ and $\Omega_{m}=0.03 \pm 0.05$. The matter component is small enough and matches well with our theoretical assumption that only the normal matter is required.

We comment on the possible constraints from gravitational wave observations. It is argued that in general the modified gravity models are constrained from two aspects [33]. One is the constraint of the energy loss rate from ultra high energy cosmic rays, which indicates that gravitational waves should propagate at the speed of light. The other is the observed gravitational waveforms from LIGO, which are consistent with Einstein's gravity and suggest that the gravitational wave should satisfy linear equations of motion in the weak-field limit. For our model, the Bianchi identity leads to $0 \equiv \nabla^{\mu} G_{\mu \nu}=\kappa_{4} \nabla^{\mu} T_{\mu \nu}+\kappa_{4} \nabla^{\mu}\langle\mathcal{T}\rangle_{\mu \nu}$. If we do not put additional sources in the bulk, the Brown-York stress-energy tensor (2) itself is conserved $\nabla^{\mu}\langle\mathcal{T}\rangle_{\mu \nu}=0$. Thus, it is similar to the effects of particle dark matter and it does not conflict with the observations from LIGO so far [34].

\section{Summary}

In summary, we construct a model of the dark fluid in our universe, which originates from the holographic stress-energy tensor $\langle\mathcal{T}\rangle_{\mu \nu}^{d}$ of higher dimensional spacetime. The toy hEDU model on a de-Sitter screen in flat bulk spacetime produces one additional constraint from $\Lambda$ CDM parameterization to the components of the late-time universe. We derive the corresponding Friedmann equation and present a good fitting result with the observational data. Finally, we would like to mention the literature on modified Newtonian dynamics (MOND) from a braneworld picture $[35,36]$, as well as the holographic big bang model in $[37,38]$ which describes the early universe with a 3-brane out of a collapsing star in (4+1)- dimensional bulk. These concepts are all related to our setups in the hEDU model. These models propose a possible origin of dark matter and dark energy and shed light on the underlying construction of the 

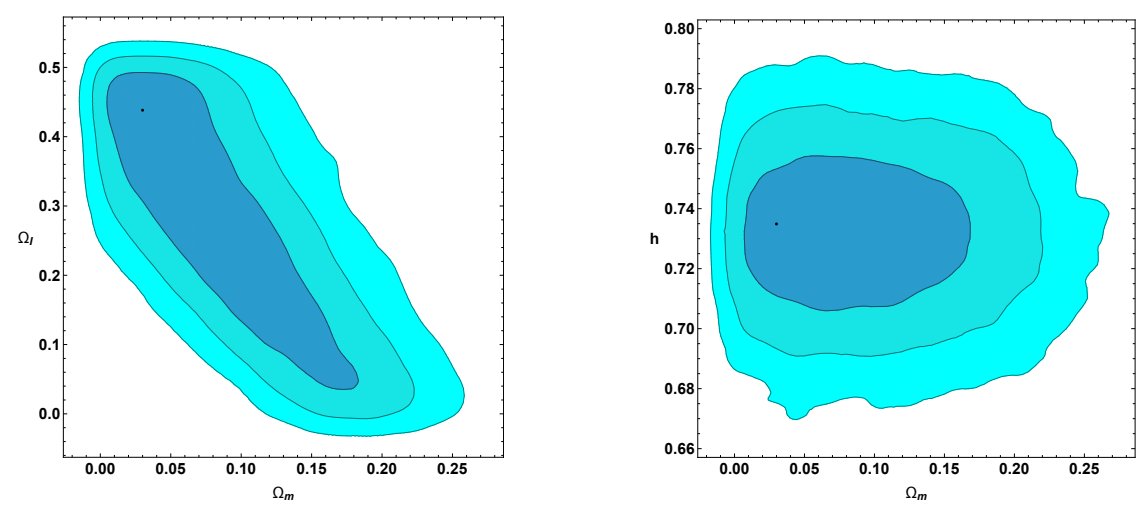

Figure 3: The 1-3 $\sigma$ confidence contours for various parameters in the hEDU model, $\Omega_{m}, \Omega_{I}, h=H_{0} /\left(100 \mathrm{~km} \mathrm{~s}^{-1} \mathrm{Mpc}^{-1}\right)$, with figures taken from [6]. It is based on the MCMC sampling analysis with the observational data of Type Ia supernovae (SNIa) and the direct measurement of Hubble constant $H_{0}$.

universe.

Acknowledgement - We thank Sunly Khimphun, Bum-Hoon Lee and Sichun Sun for the collaboration on relevant topics. R. -G. Cai was supported by the National Natural Science Foundation of China (No.11690022, No.11435006, No.11647601,No. 11851302, and No. 11821505), Strategic Priority Research Program of CAS (No. XDB23030100), Key Research Program of Frontier Sciences of CAS; G. Tumurtushaa was supported by the Institute for Basic Science (IBS) under the project code(IBS-R018-D1); Y. -L. Zhang was supported by Grant-in-Aid for JSPS international research fellow(18F18315).

\section{References}

[1] T. M. C. Abbott et al. [DES Collaboration], "Dark Energy Survey year 1 results: Cosmological constraints from galaxy clustering and weak lensing,” Phys. Rev. D 98, no. 4, 043526 (2018) [arXiv:1708.01530 [astroph.CO]].

[2] A. G. Riess, S. Casertano, W. Yuan, L. M. Macri and D. Scolnic, "Large Magellanic Cloud Cepheid Standards Provide a 1\% Foundation for the Determination of the Hubble Constant and Stronger Evidence for Physics Beyond LambdaCDM," Astrophys. J. 876, no.1, 85 (2019) arXiv:1903.07603 [astro-ph.CO].

[3] E. P. Verlinde, "Emergent Gravity and the Dark Universe," SciPost Phys. 2, 016 (2017) [arXiv:1611.02269 [hep-th]].

[4] E. P. Verlinde, "On the Origin of Gravity and the Laws of Newton," JHEP 1104, 029 (2011) [arXiv:1001.0785 [hep-th]].

[5] R. G. Cai, S. Sun and Y. L. Zhang, "Emergent Dark Matter in Late Time Universe on Holographic Screen," JHEP 1810, 009 (2018) [arXiv:1712.09326 [hep-th]].

[6] R. G. Cai, S. Khimphun, B. H. Lee, S. Sun, G. Tumurtushaa and Y. L. Zhang, "Emergent Dark Universe and the Swampland Criteria," Phys. Dark Univ. 26, 100387 (2019) arXiv:1812.11105 [hep-th].

[7] J. D. Brown and J. W. York, Jr., "Quasilocal energy and conserved charges derived from the gravitational action," Phys. Rev. D 47, 1407 (1993) [gr-qc/9209012].

[8] I. Bredberg, C. Keeler, V. Lysov and A. Strominger, "Wilsonian Approach to Fluid/Gravity Duality," JHEP 1103, 141 (2011) [arXiv:1006.1902 [hep-th]].

[9] I. Bredberg, C. Keeler, V. Lysov and A. Strominger, "From Navier-Stokes To Einstein," JHEP 1207, 146 (2012) [arXiv:1101.2451 [hep-th]].

[10] R. G. Cai, L. Li and Y. L. Zhang, "Non-Relativistic Fluid Dual to Asymptotically AdS Gravity at Finite Cutoff Surface," JHEP 1107, 027 (2011) [arXiv:1104.3281 [hep-th]].

[11] D. Brattan, J. Camps, R. Loganayagam and M. Rangamani, "CFT dual of the AdS Dirichlet problem : Fluid/Gravity on cut-off surfaces,” JHEP 1112, 090 (2011) [arXiv:1106.2577 [hep-th]].

[12] R. G. Cai, L. Li, Z. Y. Nie and Y. L. Zhang, "Holographic Forced Fluid Dynamics in Non-relativistic Limit," Nucl. Phys. B 864, 260 (2012) [arXiv:1202.4091 [hep-th]]. 
[13] X. Bai, Y. P. Hu, B. H. Lee and Y. L. Zhang, "Holographic Charged Fluid with Anomalous Current at Finite Cutoff Surface in Einstein-Maxwell Gravity," JHEP 1211, 054 (2012) [arXiv:1207.5309 [hep-th]].

[14] R. G. Cai, T. J. Li, Y. H. Qi and Y. L. Zhang, "Incompressible Navier-Stokes Equations from Einstein Gravity with Chern-Simons Term,” Phys. Rev. D 86, 086008 (2012) [arXiv:1208.0658 [hep-th]].

[15] G. Compere, P. McFadden, K. Skenderis and M. Taylor, "The Holographic fluid dual to vacuum Einstein gravity," JHEP 1107, 050 (2011) [arXiv:1103.3022 [hep-th]].

[16] G. Compere, P. McFadden, K. Skenderis and M. Taylor, "The relativistic fluid dual to vacuum Einstein gravity," JHEP 1203, 076 (2012) [arXiv:1201.2678 [hep-th]].

[17] C. Eling, A. Meyer and Y. Oz, "The Relativistic Rindler Hydrodynamics," JHEP 1205, 116 (2012) [arXiv:1201.2705 [hep-th]].

[18] R. G. Cai, L. Li, Q. Yang and Y. L. Zhang, "Petrov type I Condition and Dual Fluid Dynamics," JHEP 1304, 118 (2013) [arXiv:1302.2016 [hep-th]].

[19] R. G. Cai, Q. Yang and Y. L. Zhang, "Petrov type I Spacetime and Dual Relativistic Fluids," Phys. Rev. D 90, no. 4, 041901 (2014) [arXiv:1401.7792 [hep-th]].

[20] N. Pinzani-Fokeeva and M. Taylor, “Towards a general fluid/gravity correspondence,” Phys. Rev. D 91, no. 4, 044001 (2015) [arXiv:1401.5975 [hep-th]].

[21] R. G. Cai, Q. Yang and Y. L. Zhang, "Petrov type I Condition and Rindler Fluid in Vacuum Einstein-GaussBonnet Gravity," JHEP 1412, 147 (2014) [arXiv:1408.6488 [hep-th]].

[22] S. Khimphun, B. H. Lee, C. Park and Y. L. Zhang, "Rindler Fluid with Weak Momentum Relaxation," JHEP 1801, 058 (2018) [arXiv:1705.05078 [hep-th]].

[23] P. A. R. Ade et al. [Planck Collaboration], "Planck 2015 results. XIII. Cosmological parameters," Astron. Astrophys. 594, A13 (2016) [arXiv:1502.01589 [astro-ph.CO]].

[24] P. Binetruy, C. Deffayet and D. Langlois, "Nonconventional cosmology from a brane universe," Nucl. Phys. B 565, 269 (2000) [hep-th/9905012].

[25] R. Dick, "Brane worlds," Class. Quant. Grav. 18, no. 17, R1 (2001) [hep-th/0105320].

[26] R. H. Price and K. S. Thorne, "Membrane Viewpoint on Black Holes: Properties and Evolution of the Stretched Horizon," Phys. Rev. D 33, 915 (1986).

[27] M. Parikh and F. Wilczek, "An action for black hole membranes," Phys. Rev. D 58, 064011 (1998)

[28] G. R. Dvali, G. Gabadadze and M. Porrati, "4-D gravity on a brane in 5-D Minkowski space," Phys. Lett. B 485, 208 (2000) [hep-th/0005016].

[29] C. Deffayet, "Cosmology on a brane in Minkowski bulk," Phys. Lett. B 502, 199 (2001) [hep-th/0010186].

[30] S. Mukohyama, "Brane world solutions, standard cosmology, and dark radiation," Phys. Lett. B 473, 241 (2000) [hep-th/9911165].

[31] A. Lue, "The phenomenology of Dvali-Gabadadze-Porrati cosmologies," Phys. Rept. 423, 1 (2006) [astro$\mathrm{ph} / 0510068]$.

[32] A. G. Riess et al., "A 2.4\% Determination of the Local Value of the Hubble Constant," Astrophys. J. 826, no. 1, 56 (2016) [arXiv:1604.01424 [astro-ph.CO]].

[33] P. M. Chesler and A. Loeb, "Constraining Relativistic Generalizations of Modified Newtonian Dynamics with Gravitational Waves," Phys. Rev. Lett. 119, 031102 (2017) [arXiv:1704.05116 [astro-ph.HE]].

[34] M. A. Green, J. W. Moffat and V. T. Toth, "Modified Gravity (MOG), the speed of gravitational radiation and the event GW170817/GRB170817A," Phys. Lett. B 780, 300 (2018) [arXiv:1710.11177 [gr-qc]].

[35] C. M. Ho, D. Minic and Y. J. Ng, “Cold Dark Matter with MOND Scaling," Phys. Lett. B 693, 567 (2010) [arXiv:1005.3537 [hep-th]].

[36] M. Milgrom, "MOND from a brane-world picture," arXiv:1804.05840 [gr-qc].

[37] R. Pourhasan, N. Afshordi and R. B. Mann, "Out of the White Hole: A Holographic Origin for the Big Bang," JCAP 1404, 005 (2014) [arXiv:1309.1487 [hep-th]].

[38] N. Altamirano, E. Gould, N. Afshordi and R. B. Mann, "Cosmological Perturbations in the 5D Holographic Big Bang Model," arXiv:1703.00954 [astro-ph.CO]. 